\title{
Right ventricular contractility in systemic sclerosis-associated and idiopathic pulmonary arterial hypertension
}

\author{
M.J. Overbeek*, J-W. Lankhaar*,\#, N. Westerhof*,", A.E. Voskuyl', \\ A. Boonstra*, J.G.F. Bronzwaer ${ }^{\S}$, K.M.J. Marques ${ }^{\S}$, E.F. Smit*, \\ B.A.C. Dijkmans ${ }^{+}$and A. Vonk-Noordegraaf*
}

ABSTRACT: Since systemic sclerosis (SSc) also involves the heart, the aim of the present study was to evaluate possible differences in right ventricular (RV) pump function between SScassociated pulmonary arterial hypertension (PAH; SScPAH) and idiopathic PAH (IPAH).

In 13 limited cutaneous SScPAH and 17 IPAH patients, RV pump function was described using the pump function graph, which relates mean RV pressure $\left(\bar{P}_{\mathrm{RV}}\right)$ and stroke volume index (SVI). Differences in pump function result in shift or rotation of the pump function graph. $\bar{P}_{\mathrm{RV}}$ and SVI were measured using standard catheterisation. The hypothetical isovolumic $\bar{P}_{\mathrm{RV}}\left(\bar{P}_{\mathrm{RV}}\right.$,iso) was estimated using a single-beat method. The pump function graph was approximated by a parabola: $\bar{P}_{\mathrm{RV}}=\overline{\mathrm{P}}_{\mathrm{RV}}$,iso[1-(SVI/SVImax $)^{2}$ ], where SVImax is the hypothetical maximal SVI at zero $\bar{P}_{\mathrm{RV}}$, enabling calculation of SVImax.

There were no differences in SVI and SVImax. Both $\bar{P}_{\mathrm{RV}}$ and $\bar{P}_{\mathrm{RV}}$,iso were significantly lower in SScPAH than in IPAH ( $\bar{P}_{\mathrm{RV}} 30.7 \pm 8.5$ versus $41.2 \pm 9.4 \mathrm{mmHg} ; \bar{P}_{\mathrm{RV}}$, iso $43.1 \pm 12.4$ versus $53.5 \pm 10.0 \mathrm{mmHg}$ ). Since higher pressures were found at similar SVI, the difference in the pump function graph results from lower contractility in SScPAH than in IPAH.

Right ventricular contractility is lower in systemic sclerosis-associated pulmonary arterial hypertension than in idiopathic pulmonary arterial hypertension.

KEYWORDS: Myocardial contraction, pump function graph, right ventricular function, right ventricular pressure, stroke volume

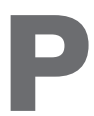

atients with systemic sclerosis (SSc) are at high risk of developing pulmonary arterial hypertension $(\mathrm{PAH})$, with estimated prevalences ranging $7.9-12 \%[1,2]$. SSc-associated PAH (SScPAH) patients exhibit a higher risk of death than those with idiopathic PAH (IPAH), as demonstrated by KAWUT et al. [3] and FISHER et al. [4]. These differences have not been explained satisfactorily to date but comorbidity due to the systemic character of SSc, age-related factors due to the later disease onset of PAH in SSc and differences in pulmonary vasculopathy [5] may all play a role. In addition, the SScPAH patients described by KAWUT et al. [3] and FisHER et al. [4] showed a higher mortality despite a similar or lower pulmonary vascular resistance (PVR) at baseline. This suggests an inferior ability of the SScPAH right ventricle (RV) to adapt to the arterial load compared with the IPAH RV. It was, therefore, hypothesised that RV contractility is impaired in SScPAH compared with IPAH.
In order to test this hypothesis, possible differences in cardiac output (CO), RV pressure (PRV) and arterial load, in terms of PVR and pulmonary arterial compliance, were first characterised between the two groups. Following this, the RV pump function of both groups was characterised using the pump function graph [6], describing the cardiac pumping ability in terms of the relationship between mean PRV ( $\left.\bar{P}_{\mathrm{RV}}\right)$ and CO.

\section{MATERIAL AND METHODS}

\section{Study design}

Patients diagnosed with SScPAH $(n=13)$ or IPAH $(n=17)$ at the VU University Medical Centre (Amsterdam, the Netherlands) between July 2000 and July 2006 were included, and their PRV and pulmonary artery pressure $(\mathrm{Ppa})$ waveforms recorded and digitally stored during standard right heart catheterisation. The present study was approved by the Institutional Review Board on Research Involving Human Subjects of the VU University Medical Centre.

\section{AFFILIATIONS}

*Depts of Pulmonary Diseases,

\#Physics and Medical Technology,

"Physiology,

${ }^{+}$Rheumatology, and

${ }^{\S}$ Cardiology, Institute for

Cardiovascular Research. VU

University Medical Centre, Vrije

University, Amsterdam, The

Netherlands.

CORRESPONDENCE

A. Vonk-Noordegraaf

Dept of Pulmonary Diseases

VU University Medical Centre

De Boelelaan 1117

$1081 \mathrm{HV}$

P.0. Box 7057

1007 MB Amsterdam

The Netherlands

Fax: 31204444328

E-mail: A.Vonk@VUmc.nl

Received:

October 142007

Accepted after revision:

January 022008

SUPPORT STATEMENT

J-W. Lankhaar was supported by the Netherlands Heart Foundation (The

Hague, the Netherlands; grant

NHS2003B274).

STATEMENT OF INTEREST

Statements of interest for A. Boonstra and A. Vonk-Noordegraaf can be found at www.erj.ersjournals.com/ misc/statements.shtml

European Respiratory Journal

Print ISSN 0903-1936

Online ISSN 1399-3003 


\section{Methods}

Pulmonary hypertension was confirmed by a mean $P_{\mathrm{pa}}\left(\bar{P}_{\mathrm{pa}}\right)$ of $>25 \mathrm{mmHg}$ at rest and a pulmonary capillary wedge pressure (Ppcw) of $<15 \mathrm{mmHg}$. All catheterisations were baseline measurements. Additional clinical diagnostic work-up was performed according to a standard diagnostic protocol [7] in order to exclude other causes of pulmonary hypertension. The diagnosis of SSc was based on the classification criteria proposed by LeRoY et al. [8].

Pulmonary function testing (Vmax 229 and 6200; SensorMedics, Yorba Linda, CA, USA) and high-resolution computed tomography (HRCT; CT Somatom Plus 4; Siemens, Erlangen, Germany) were used to exclude underlying fibrotic lung disease as a cause of pulmonary hypertension.

\section{Analysis}

Haemodynamic parameters

CO was calculated using the Fick method and PVR from $\left(\bar{P}_{\text {pa- }}\right.$ $\left.P_{\mathrm{pcw}}\right) / \mathrm{CO}$. The PRV waveform was averaged to obtain $\bar{P}_{\mathrm{RV}}$. Stroke volume index (SVI) was calculated from cardiac index (CI) divided by cardiac frequency. Total pulmonary arterial compliance was calculated from stroke volume divided by pulse pressure $[9,10]$. Pulse pressure was calculated from systolic minus diastolic Ppa. Pressure measurements were recorded digitally at a sampling frequency of $250 \mathrm{~Hz}$ using a customised LabVIEW data acquisition system (National Instruments Netherlands, Woerden, the Netherlands).

\section{Pump function graph}

For the characterisation of RV pump function, a pump function graph was constructed. The pump function graph describes pump function quantitatively by the relationship between $\bar{P}_{\mathrm{RV}}$ and CO. Experimentally, this relationship has been determined by making the heart beat against a series of different arterial loads. Here SVI was used instead of $\mathrm{CO}$ in order to avoid

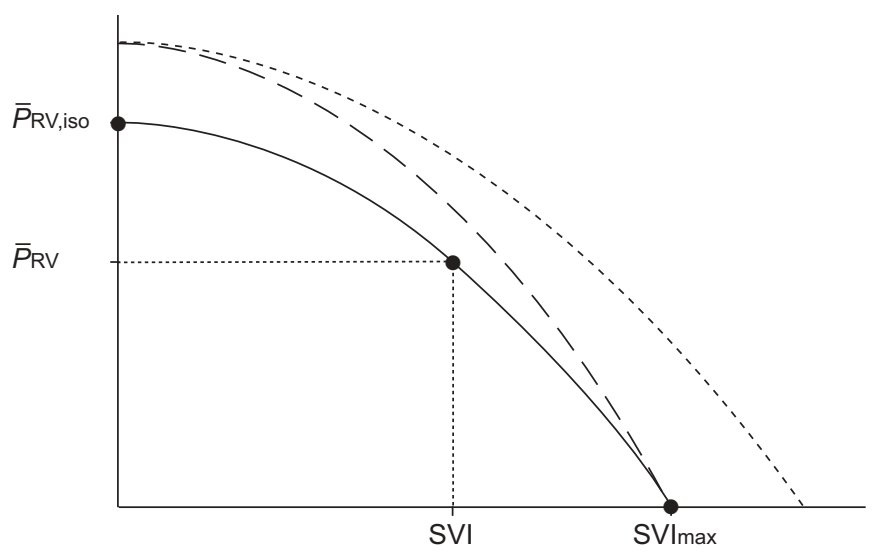

FIGURE 1. Schematically drawn ventricular pump function graph (also applicable to the left ventricle), relating mean ventricular output (stroke volume index (SVI)) and mean right ventricular pressure ( $\left.\bar{P}_{\mathrm{RV}}\right)$, and characterising the heart as a pump. —_- reference; ------: increased filling; - - - -: increased contractility. Alteration in contractility results in a rotation around the intercept on the output axis (hypothetical maximal SVI at zero $\bar{P}_{\mathrm{RV}}\left(\mathrm{SV} \mathrm{Imax}_{\mathrm{m}}\right)$ ), whereas increased filling increases both isovolumic $\bar{P}_{\mathrm{RV}}\left(\bar{P}_{\mathrm{RV}}\right.$, iso $)$ and SVImax. The three data points shown are the $\bar{P}_{\mathrm{RV}}$,iso, at zero SVI, constructed using a single-beat method, the measured $\bar{P}_{\mathrm{RV}}$ and mean SVI (working point), and the (derived) maximum output, SVImax. possible confounding effects of differences in cardiac frequency and normalise for body size. A pump function graph demonstrating the effects of alterations in diastolic filling and contractility is shown schematically in figure 1 .

From the digitally recorded PRV waveform and SVI, it was possible to obtain individual pump function graphs, which were approximated by a parabola [11]:

$$
\left.\left.\bar{P}_{\mathrm{RV}}=\bar{P}_{\mathrm{RV}} \text {,iso[1-(SVI/SVImax }\right)^{2}\right]
$$

where $\bar{P}_{\mathrm{RV}}$ is the (time) average of the PRV waveform, $\bar{P}_{\mathrm{RV}}$,iso the average of the PRV waveform of an isovolumic beat and SVImax the intercept with the SVI axis, i.e. the hypothetical maximal SVI at zero $\bar{P}_{\mathrm{RV}}$. The $\bar{P}_{\mathrm{RV}}$ and SVI were measured at catheterisation to give the working point (fig. 1).

Subsequently, an estimate of the isovolumic PRV waveform, $\bar{P} R V$,iso, was obtained using the single-beat method originally proposed by SuNAGAWA et al. [12], and validated for the RV by BRIMIOULLE et al. [13]. Its mean value is $\bar{P}_{\mathrm{RV}}$,iso, and, since SVI is zero by definition for an isovolumic beat, this yielded the second point of the pump function graph. Finally, SVImax was obtained by reorganising the previous equation:

$$
\text { SVImax }=S V I / \sqrt{1-\bar{P}_{\mathrm{RV}} / \bar{P}_{\mathrm{RV}} \text {,iso }}
$$

and inserting the measured SVI and $\bar{P}_{\mathrm{RV}}$, and the calculated $\bar{P}_{\mathrm{RV}}$,iso. Since SVImax represents the intercept on the SVI axis, this yielded the third point of the graph. The three points are indicated in figure 1.

The single-beat method assumes that the pressure waveform of an isovolumic beat can be described by a sinusoidal function and that it can be obtained by a least-squares fit to the isovolumic phases of the PRV waveform of the ejecting beat. Isovolumic contraction was assumed to start at the minimum $P$ RV before the steep rise in pressure (R-wave of ECG), and to end when $P$ RV reached diastolic $P$ pa. The isovolumic relaxation period was defined as lasting from pulmonary artery valve closure, identified by overlaying the $P$ pa waveform over the $P \mathrm{RV}$ waveform, until the $P \mathrm{RV}$ reached the diastolic pressure from which the isovolumic contraction calculations were started. A schematic example is shown in figure 2. Before the analysis, underdamping catheter artefacts were removed from the pressure waveforms using a Butterworth filter (cut-off frequency of $10 \mathrm{~Hz}$ ), and several cardiac cycles were averaged to obtain an average pressure waveform.

Statistics

Group-averaged pump function graphs were obtained by averaging isovolumic PRV and SVImax. Unpaired t-tests and Mann-Whitney U-tests were performed to compare data from both groups.

All data are presented as mean \pm SD in tables and as mean \pm SEM or median (interquartile range) in figures. A p-value of $<0.05$ was considered significant.

\section{RESULTS}

\section{General patient characteristics}

General patient characteristics are given in table 1. Patients with SScPAH were significantly older, with a mean age difference of $27 \mathrm{yrs}$. The SScPAH group comprised $100 \%$ female patients, whereas $77 \%$ of the patients in the IPAH 


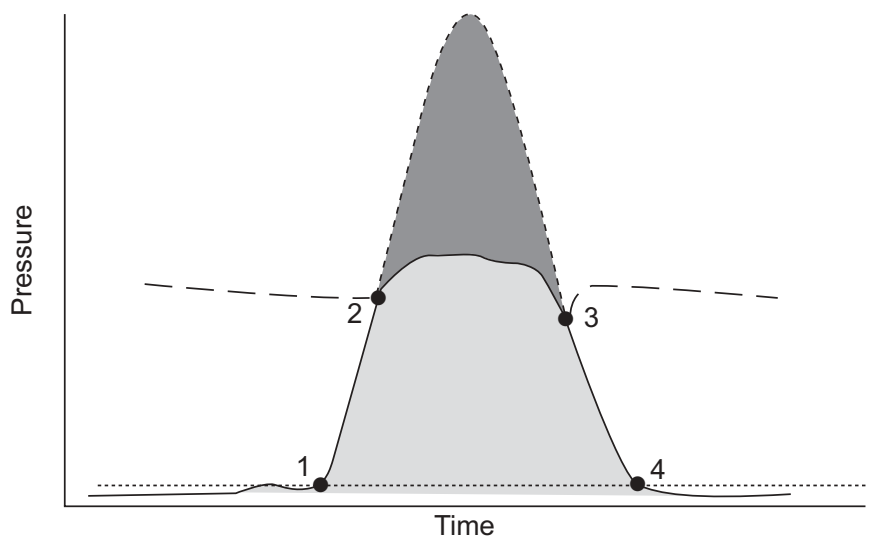

FIGURE 2. Schematic example of the derivation of an isovolumic beat. Isovolumic contraction is assumed to start at the minimum right ventricular pressure (PRV; - ) , before the steep rise in pressure (1), and to end when it reaches diastolic pulmonary arterial pressure (Ppa; - - - ; 2). The isovolumic relaxation period is defined as lasting from pulmonary artery valve closure (3), identified from deviation of $P \mathrm{RV}$ and $P \mathrm{pa}$, until the $P \mathrm{RV}$ reaches the diastolic pressure (4). The area under the PRV curve is used to calculate mean PRV ( $\left.\overline{P R V}_{\mathrm{RV}} \square\right)$. -------: derivation of isovolumic PRV; the total area under this line is used to calculate isovolumic $\bar{P}_{\mathrm{RV}}$ ( $\overline{\mathrm{PRV}, \text { iso; }}$ - $)$.

group were female. All of the SScPAH patients included suffered from the limited cutaneous form of SSc (lcSSc).

The 6-min walking distance did not differ significantly between the groups, and neither did mixed venous oxygen saturation or N-terminal-pro-B-type natriuretic peptide (NTproBNP) levels. Evaluation of pulmonary function showed that pulmonary gas exchange in $\mathrm{SSCPAH}$ patients, quantified by the transfer factor of the lung for carbon monoxide, was significantly lower than in IPAH patients, in agreement with previously reported values [14, 15]. Mean total lung capacity and evaluation by HRCT in the SSCPAH group indicated that the pulmonary hypertension cannot be explained by severe pulmonary fibrosis. HRCT showed a typical pattern of pulmonary fibrosis in the dorsobasal lung fields in seven of the $13 \mathrm{SScPAH}$ patients; these patients had a total lung capacity of $>70 \%$ of the predicted value and arterial oxygen saturations of $\geqslant 92 \%$.

\section{Haemodynamic invasive parameters and pump function graphs}

Haemodynamic parameters are listed in table 2. All patients had PVRs of $>240 \mathrm{dyn} \cdot \mathrm{s} \cdot \mathrm{cm}^{-5}$. A significantly lower $\bar{P}$ pa was found in the SScPAH group than in the IPAH group. The $\bar{P}_{\mathrm{RV}}$ was significantly lower in the SScPAH group than in the IPAH group, whereas the SVI was not significantly different (fig. 3). There was no significant difference in total arterial load, since neither PVR nor total arterial compliance differed between the groups (fig. 4).

In order to evaluate cardiac pump function, pump function graphs were constructed as described above. Examples of individual pump function graphs of an SSCPAH patient and an IPAH patient are depicted in figure 5. The averaged pump function graphs are shown in figure $6 . \bar{P} \mathrm{RV}$,iso was found to be significantly lower in SScPAH patients than in IPAH patients.

\begin{tabular}{|c|c|c|c|}
\hline & SScPAH & IPAH & p-value \\
\hline Subjects $\mathbf{n}$ & 13 & 17 & \\
\hline Age yrs & $68.6 \pm 12.4$ & $41.9 \pm 16.0$ & $<0.001$ \\
\hline Females & $13(100)$ & $13(77)$ & $<0.001^{+}$ \\
\hline \multicolumn{4}{|l|}{ Cutaneous SSc } \\
\hline Limited $^{\#}$ & $13(100)$ & & \\
\hline Diffuse $^{\#}$ & $0(0)$ & & \\
\hline \multicolumn{4}{|l|}{ Duration yrs } \\
\hline SSc disease & $9.6 \pm 6.8^{\S}$ & & \\
\hline Raynaud phenomenon & $18 \pm 15$ & & \\
\hline ACA/anti-Scl-70/anti-U1-RNP & $7 / 1 / 1(50 / 7 / 7)$ & & \\
\hline Body surface area $\mathrm{m}^{2}$ & $1.7 \pm 0.2$ & $1.9 \pm 0.3$ & 0.005 \\
\hline \multicolumn{4}{|l|}{ ABP $\mathrm{mmHg}$} \\
\hline Systolic & $129 \pm 22$ & $119 \pm 23$ & 0.49 \\
\hline Diastolic & $74 \pm 13$ & $73 \pm 11$ & 0.78 \\
\hline 6-min walking distance $\mathrm{m}$ & $277 \pm 116$ & $358 \pm 110$ & 0.08 \\
\hline $\mathrm{Sv}, \mathrm{O}_{2} \%$ & $62.0 \pm 6.5$ & $63.5 \pm 6.0$ & 0.68 \\
\hline NT-proBNP pg.mL ${ }^{-1}$ & $3546 \pm 3035^{f}$ & $1384 \pm 1160^{\# \#}$ & 0.24 \\
\hline TLC \% pred & $89.9 \pm 16.6$ & $99.9 \pm 11.8$ & 0.26 \\
\hline$T \mathrm{~L}, \mathrm{CO} \%$ & $42.8 \pm 12.6$ & $65.7 \pm 16.1$ & 0.002 \\
\hline
\end{tabular}

Data are presented as mean \pm SD or $n(\%)$, unless otherwise indicated. SScPAH systemic sclerosis (SSc)-associated pulmonary arterial hypertension (PAH); IPAH: idiopathic PAH; ACA: anticentromere antibody; Scl: scleroderma; RNP: ribonucleoprotein; $\mathrm{ABP}$ : arterial blood pressure; $\mathrm{Sv}, \mathrm{O}_{2}$ : mixed venous oxygen saturation; NT-proBNP: N-terminal-pro-B-type natriuretic peptide; TLC: total lung capacity; \% pred: \% predicted; $T L, C O$ : transfer factor of the lung for carbon monoxide. ${ }^{*}$ : as defined in [8]; ${ }^{\bullet}$ : since first non-Raynaud symptom; ${ }^{+}$: Chisquared statistic; ${ }^{\S}: \mathrm{n}=12 ;{ }^{\prime}: \mathrm{n}=10 ;{ }^{\# \#}: \mathrm{n}=9.1 \mathrm{mmHg}=0.133 \mathrm{kPa}$.

SVImax did not differ between the two groups. Thus, compared with the SScPAH patients, the IPAH patients demonstrated a higher pump function graph, rotated around the same point of the horizontal axis intercept, i.e. SVImax.

\section{DISCUSSION}

In the present study, cardiac pump function was compared between SScPAH and IPAH patients using the relationship between $\bar{P}_{\mathrm{RV}}$ and SVI. These variables were obtained using standard right heart catheterisation and Fick $\mathrm{CO}$ measurements. Lower values were found for $\bar{P}_{\mathrm{RV}}$ in the SScPAH group than in the IPAH group, whereas SVIs were not significantly different. Analysis of the arterial system in terms of PVR and total arterial compliance showed no difference in arterial load between the two groups. On the basis of these data, it is concluded that right heart pump function differs between the $\mathrm{SScPAH}$ and IPAH groups.

These haemodynamic differences between SScPAH and IPAH patients are in agreement with those described by FISHER et al. [4]. Although they did not evaluate cardiac function, their haemodynamic data showed a similar pattern to the present one, consisting of a similar CI at lower $\bar{P}$ pa. In their study, PVR was significantly lower in SScPAH than in IPAH, in contrast to the comparable PVRs between the groups in the present study cohort. This supports the present data; despite lower afterload, SScPAH patients were unable to generate a 
TABLE 2 Haemodynamics and pump function graph data

\begin{tabular}{|c|c|c|c|}
\hline & SScPAH & IPAH & p-value \\
\hline Subjects n & 13 & 17 & \\
\hline Cardiac frequency beats $\cdot \min ^{-1}$ & $82.4 \pm 13.0$ & $86.7 \pm 13.8$ & 0.43 \\
\hline $\bar{P}_{\text {RA }} \mathrm{mmHg}$ & $6 \pm 4$ & $8 \pm 5$ & 0.56 \\
\hline $\bar{P}_{R V} \mathbf{m m H g}$ & $31 \pm 9$ & $41 \pm 9$ & 0.006 \\
\hline $\bar{P}_{\mathrm{RV}, \text { iso }} \mathrm{mmHg}$ & $43 \pm 12$ & $54 \pm 10$ & 0.043 \\
\hline PRV,sys mmHg & $43 \pm 12$ & $60 \pm 10$ & $<0.0001$ \\
\hline PRV,dias $\mathrm{mmHg}$ & $11 \pm 5$ & $14 \pm 7$ & 0.48 \\
\hline$\overline{\mathrm{P}} \mathrm{pa}, \mathrm{mmHg}$ & $44 \pm 10$ & $60 \pm 10$ & $<0.0001$ \\
\hline Ppa,sys mmHg & $70 \pm 14$ & $97 \pm 19$ & $<0.0001$ \\
\hline Ppa,dias $\mathrm{mmHg}$ & $26 \pm 6$ & $37 \pm 8$ & $<0.0001$ \\
\hline Ppcw mmHg & $9 \pm 34$ & $8 \pm 4$ & 0.62 \\
\hline PVR dyn $\cdot s \cdot \mathrm{cm}^{-5}$ & $848 \pm 397$ & $1079 \pm 433$ & 0.13 \\
\hline Compliance $\mathrm{mL} \cdot \mathrm{mmHg}^{-1}$ & $1.1 \pm 0.42$ & $0.9 \pm 0.43$ & 0.30 \\
\hline $\mathrm{SVI} \mathrm{mL} \cdot \mathrm{m}^{-2}$ & $27.1 \pm 7.3$ & $26.7 \pm 7.6$ & 0.84 \\
\hline SVImax $\mathrm{mL} \cdot \mathrm{m}^{-2}$ & $53.5 \pm 20.6$ & $57.5 \pm 15.9$ & 0.23 \\
\hline $\mathrm{CI} \mathrm{L} \cdot \mathrm{min}^{-1} \cdot \mathrm{m}^{-2}$ & $2.2 \pm 0.6$ & $2.3 \pm 0.6$ & 0.71 \\
\hline
\end{tabular}

Data are presented as mean \pm SD. SScPAH: systemic sclerosis-associated pulmonary arterial hypertension (PAH); IPAH: idiopathic PAH; $\bar{P}_{\text {RA: }}$ mean right atrial pressure; $\bar{P}_{\mathrm{RV}}$ : mean right ventricular pressure $(P \mathrm{RV}) ; \bar{P}_{\mathrm{RV}}$, iso: isovolumic $\bar{P} \mathrm{RV} ; P_{\mathrm{RV}}$,sys: systolic $P_{\mathrm{RV}} ; P_{\mathrm{RV}}$,dias: diastolic $P \mathrm{RV}$; $\bar{P}$ pa: mean pulmonary arterial pressure (Ppa); $P$ pa,sys: systolic $P$ pa; $P$ pa,dias: diastolic $P$ pa; $P$ pcw: pulmonary capillary wedge pressure; PVR: pulmonary vascular resistance; Compliance: total pulmonary arterial compliance; SVI: stroke volume index; SVImax: maximal $\mathrm{SVI}$ at zero $\overline{\mathrm{PRV}}$; $\mathrm{Cl}$ : cardiac index. $1 \mathrm{mmHg}=0.133 \mathrm{kPa}$

higher CI than IPAH patients. In addition, they observed a significantly higher mortality in the SScPAH group compared with the IPAH group, despite the fact that IPAH patients had higher PVRs, supporting the idea that cardiac involvement contributes to the early death in SSCPAH.

In order to characterise differences in cardiac function, a pump function graph was constructed for each patient. A pump function graph presents the pumping ability of the RV. ELZINGA and WESTERHOF $[16,17]$ have shown, in isolated cat hearts, that the pump function of the left and right heart can be described quantitatively by such a graph, which relates mean ventricular pressure with mean ventricular output. This relationship, which characterises the heart, was determined by making the heart eject against a series of different arterial loads. Changing the afterload of an individual heart moves the pressures and flows on this graph, i.e. increased load decreases $\mathrm{CO}$ and increases PRV. The pump function graph was shown to depend on cardiac frequency, ventricular filling and cardiac muscle contractility, i.e. muscle properties [6]. By using SVI instead of $\mathrm{CI}$, the effects of differences in cardiac frequency were avoided in the present study. As schematically shown in figure 1, an increase in end-diastolic volume causes a parallel outward shift of the pump function graph, whereas increased contractility results in a rotation about the intercept on the SVI axis. Thus the present data, by showing this rotation of the pump function graph to lower pressures but with a comparable intercept on the abscissa in SScPAH compared with IPAH, indicate lower contractility in the SScPAH group.
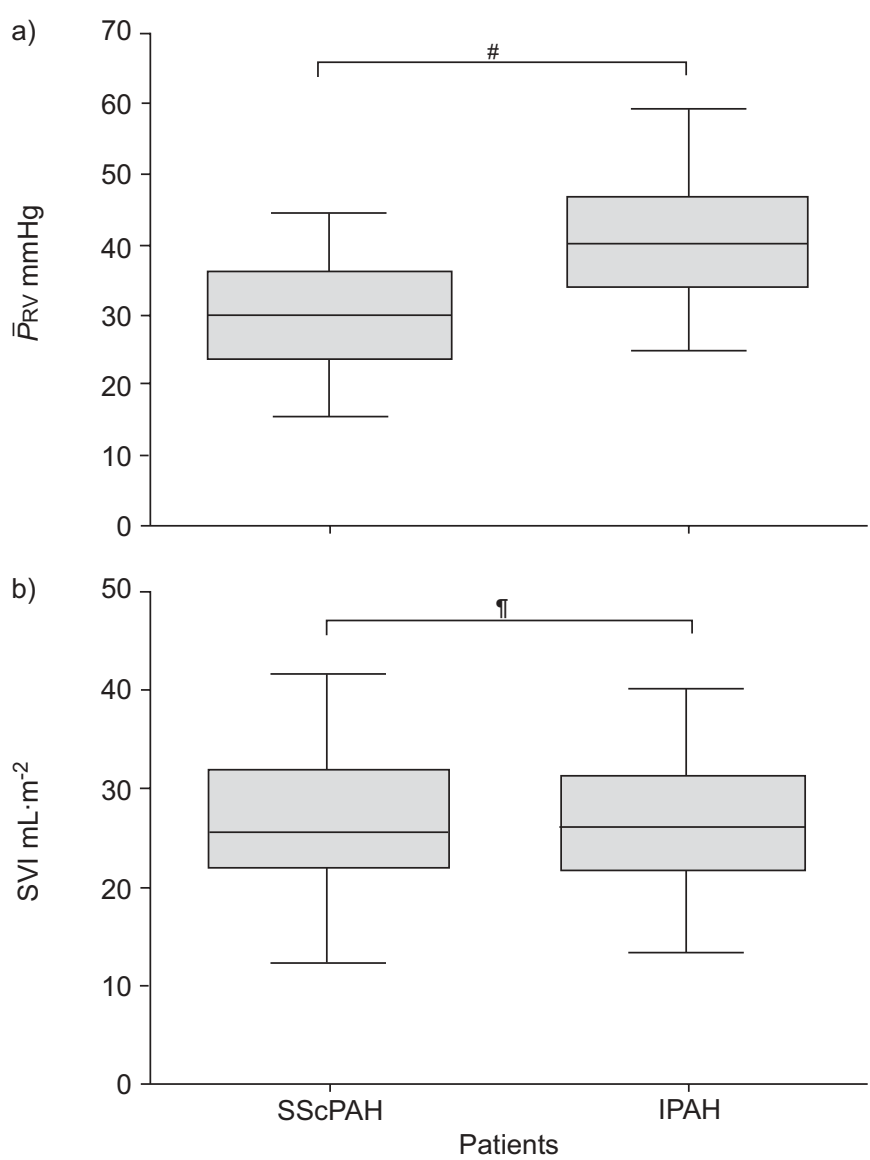

FIGURE 3. Boxplot showing a) mean right ventricular pressure ( $\left.\bar{P}_{\mathrm{RV}}\right)$, and b) stroke volume index (SVI) in systemic-sclerosis-associated pulmonary arterial hypertension (SSCPAH; $n=13$ ) and idiopathic pulmonary arterial hypertension (IPAH; $n=17)$. Boxes represent median and interquartile range; vertical bars represent range. ${ }^{*}: p=0.006 ; ?: p=0.84 .1 \mathrm{mmHg}=0.133 \mathrm{kPa}$.

The single-beat method was used to derive isovolumic PRV from a measured PRV of an ejecting beat. SUNAGAWA et al. [12] found that, for the left ventricle, there is a correlation between the isovolumic pressure observed during an isovolumic beat and the isovolumic pressure that is predicted by sine wave extrapolation from the isovolumic parts of an ejecting beat. BRIMIOULLE et al. [13] showed that this single-beat method can also be used for the RV. In the present study, this method was used to predict the $\bar{P}_{\mathrm{RV}}$,iso in individual patients in order to be able to describe a full pump function graph.

Since PVR and compliance did not differ, it was concluded that the difference in PRV between SSCPAH and IPAH hearts is based on differences in the performance of the heart itself.

The advantage of the use of the ventricular pump function graph is that it is based on standard catheterisation measurements, Fick CO and PRV. However, this method still requires further validation in humans. Validation of the pump function graph method could be performed by the evaluation of difference in contractility between the SScPAH and IPAH groups by construction of a series of RV pressure-volume loops during the temporary occlusion of the inferior vena cava, measured by means of a conductance catheter [18]. However, 

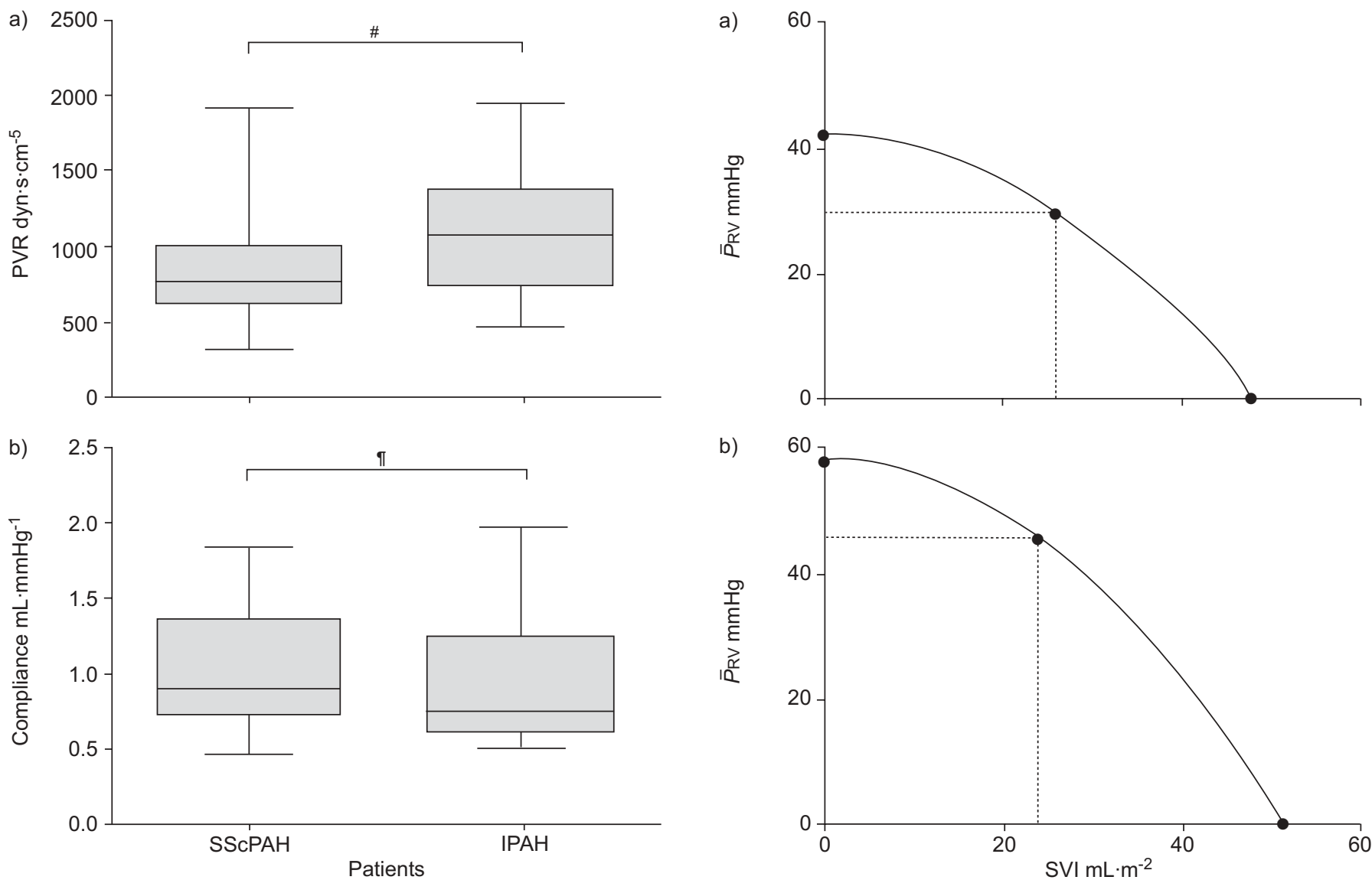

FIGURE 4. Boxplot showing the arterial load. a) Pulmonary vascular resistance (PVR), and b) pulmonary arterial compliance in systemic-sclerosis-associated pulmonary arterial hypertension (SScPAH; $n=13$ ) and idiopathic pulmonary arterial hypertension (IPAH; $n=17$ ). Boxes represent median and interquartile range; vertical bars represent range. ${ }^{\#}: p=0.12 ;{ }^{\circ}: p=0.30 .1 \mathrm{mmHg}=0.133 \mathrm{kPa}$.

this is an intervention with substantial patient burden. Moreover, volume measurement using the conductance catheter in the RV is a possibility [19], but still not sufficiently evaluated. Another method would be to simultaneously measure PRV by right heart catheterisation and RV volume by magnetic resonance imaging analysis; this method also requires further validation [20].

The lower contractility in SScPAH might be explained in several ways. Myocardial fibrosis, as well as intramyocardial coronary vessel involvement, is known to affect the ventricles in SSc [21, 22]. FERNANDES et al. [23] analysed endomyocardial biopsy specimens from SSc patients with both the lcSSc and diffuse cutaneous SSc (dcSSc) forms, without signs or symptoms of heart failure, and excluded patients with pulmonary or arterial hypertension, left ventricular hypertrophy and left ventricular diastolic dysfunction. They demonstrated abnormal collagen deposition in $94 \%$ of cases. Impaired contractility of hearts of patients with SSCPAH might then be explained by increased levels of extracellular matrix, which might affect normal contraction of the cardiac myocytes it surrounds [24, 25]. Remodelling of the heart due to persistent elevations of ventricular developed pressure leads

FIGURE 5. Examples of pump function graphs. a) A patient with systemicsclerosis-associated pulmonary arterial hypertension (PAH); and b) a patient with idiopathic PAH. $\bar{P}$ RV: mean right ventricular pressure; SVI: stroke volume index. $1 \mathrm{mmHg}=0.133 \mathrm{kPa}$.

to changes in the amount of collagen, collagen phenotype and collagen cross-linking [26]. Cross-linking has not been investigated in the hearts of SSc patients, but is increased in skin with SSc $[27,28]$. It may be speculated that SSc myocardial tissue expresses an increased degree of collagen cross-linking that contributes to an impaired cardiac contractility.

An impaired contractility in SScPAH could also be explained by ischaemia due to vascular alterations. It has been shown that structural abnormalities of small coronary arteries or arterioles explain a reduced coronary reserve in SSc $[29,30]$.

Other underlying pathophysiological mechanisms may be found at the level of cardiac muscle per se. One of the mechanisms by which the cardiac muscle adapts to ventricular pressure overload under normal conditions is hypertrophy. However, impaired contractility of SSCPAH hearts might not be explained by an inability of the SScPAH heart to undergo hypertrophy, since the RV mass of SScPAH hearts has been shown to be comparable to that of IPAH hearts $(41.6 \pm 12.3$ $(\mathrm{n}=11)$ versus $\left.45.8 \pm 14.8 \mathrm{~g} \cdot \mathrm{m}^{-2}(\mathrm{n}=14) ; \mathrm{p}=0.51\right)$ [31]. This does not exclude the possibility that other intrinsic myocyte pathology may be responsible for impaired cardiac contractility in the SScPAH group, although information on this topic, for example regarding intrinsic myocyte abnormalities that 


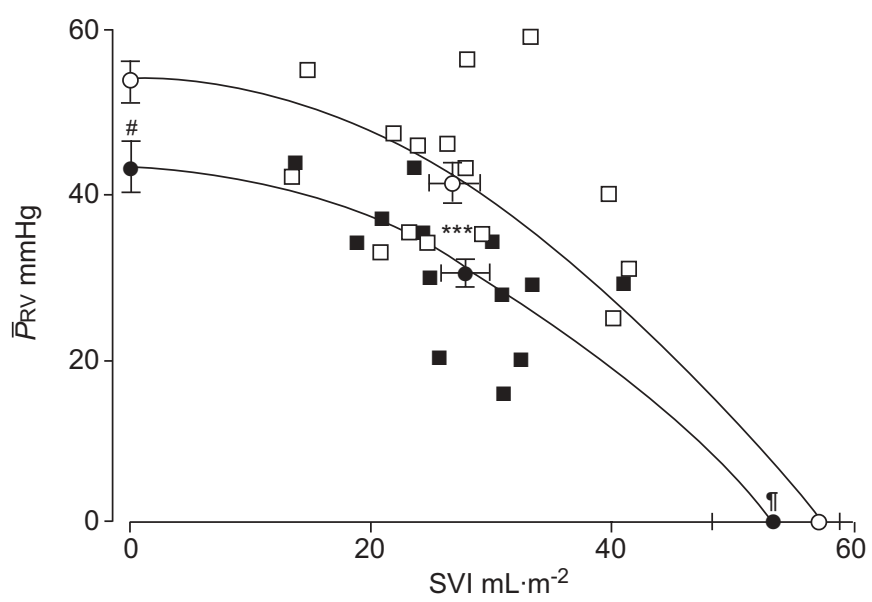

FIGURE 6. Averaged ventricular pump function graph for systemic-sclerosisassociated pulmonary arterial hypertension (SSCPAH; - and $\mathbf{\square}$; $=13$ ) and idiopathic pulmonary arterial hypertension (IPAH; $\bigcirc$ and $\square ; n=17$ ). Data are presented as mean \pm SEM for: isovolumic mean right ventricular pressure $\left(\bar{P}_{\mathrm{RV}, i s o}\right)$ at zero stroke volume index (SVI); the measured $\bar{P}_{\mathrm{RV}}$ and SVI (working point); and the (derived) maximum output, maximal SVI at zero $\bar{P}_{\mathrm{RV}}$ (SVImax). The IPAH pump function graph is located above the SScPAH pump function graph, with a rotation around the SVImax. ${ }^{*}: p=0.04 ; ": p=0.23$ versus IPAH group. $1 \mathrm{mmHg}=0.133 \mathrm{kPa}$.

may be responsible for impaired contractility in the SScPAH group, is not available. The present hypothesis of intrinsic myocardial involvement in SSCPAH patients is supported by the findings of MEUNE et al. [32], who found a decreased RV ejection fraction in patients with early SSc (both lcSSc and dcSSc), without relation to $\bar{P}$ pa.

In the present study group, the SScPAH patients were significantly older than the IPAH patients, reflecting the normal epidemiological features [15, 33]. Age differences might affect RV diastolic function, but have not been demonstrated to affect RV systolic function [34, 35]. However, in order to exclude age-related factors as the underlying explanation of the differences found in the present study, a study with age-matched SScPAH and IPAH patients may elucidate the influence of age on RV contractility in PAH. Other limitations are the weak power of the present study due to low patient numbers. Moreover, there is heterogeneity of patients, as shown by the ranges of NT-proBNP levels, PVR and compliance.

The SScPAH population consisted of patients with the lcSSc form of SSc [8], which may lead to a bias since no dcSSc patients were studied. First, patients with the dcSSc form of SSc are more likely to suffer from pulmonary fibrosis as a contributor or cause of pulmonary hypertension, and, since the present patient group consisted of lcSSc patients with no or mild fibrosis on HRCT, fibrosis was not considered as a potential cause of pulmonary hypertension. It was thus assumed that the SSc patients in the present study suffered from pulmonary hypertension caused by pre-capillary vasculopathy and, as such, are comparable with the IPAH patients. Secondly, it might be that cardiac involvement in SSc differs between dcSSc and lcSSc. As differences in this respect have not been elucidated, it is difficult to extrapolate the present findings to dcSSc, since no knowledge exists regarding differences in heart involvement and adaptation in response to elevated PVR between lcSSc and dcSSc.

In conclusion, using standard catheterisation data, it has been demonstrated that systemic sclerosis-associated pulmonary arterial hypertension patients exhibit lower cardiac contractility than idiopathic pulmonary arterial hypertension patients. Further study of right ventricular function in systemic sclerosis-associated pulmonary arterial hypertension should elucidate the underlying mechanisms.

\section{REFERENCES}

1 Hachulla E, Gressin V, Guillevin L, et al. Early detection of pulmonary arterial hypertension in systemic sclerosis: a French nationwide prospective multicenter study. Arthritis Rheum 2005; 52: 3792-3800.

2 Mukerjee D, St George D, Coleiro B, et al. Prevalence and outcome in systemic sclerosis associated pulmonary arterial hypertension: application of a registry approach. Ann Rheum Dis 2003; 62: 1088-1093.

3 Kawut SM, Taichman DB, Archer-Chicko CL, Palevsky HI, Kimmel SE. Hemodynamics and survival in patients with pulmonary arterial hypertension related to systemic sclerosis. Chest 2003; 123: 344-350.

4 Fisher MR, Mathai SC, Champion HC, et al. Clinical differences between idiopathic and scleroderma-related pulmonary hypertension. Arthritis Rheum 2006; 54: 3043-3050.

5 Dorfmuller P, Humbert M, Perros F, et al. Fibrous remodeling of the pulmonary venous system in pulmonary arterial hypertension associated with connective tissue diseases. Hum Pathol 2007; 38: 893-902.

6 Westerhof N, Stergiopulos N, Noble MIM. Pump function graph. In: Snapshots of Hemodynamics. An Aid for Clinical Research and Graduate Education. 1st Edn. New York, Springer Science and Business Media, Inc., 2005; pp. 63-70.

7 Barst RJ, McGoon M, Torbicki A, et al. Diagnosis and differential assessment of pulmonary arterial hypertension. J Am Coll Cardiol 2004; 43: Suppl. 12, 40S-47S.

8 LeRoy EC, Black C, Fleischmajer R, et al. Scleroderma (systemic sclerosis): classification, subsets and pathogenesis. J Rheumatol 1988; 15: 202-205.

9 Mahapatra S, Nishimura RA, Sorajja P, Cha S, McGoon MD. Relationship of pulmonary arterial capacitance and mortality in idiopathic pulmonary arterial hypertension. J Am Coll Cardiol 2006; 47: 799-803.

10 Chemla D, Hebert JL, Coirault C, et al. Total arterial compliance estimated by stroke volume-to-aortic pulse pressure ratio in humans. Am J Physiol 1998; 27: H500-H505.

11 van den Horn GJ, Westerhof N, Elzinga G. Optimal power generation by the left ventricle. A study in the anesthetized open thorax cat. Circ Res 1985; 56: 252-261.

12 Sunagawa K, Yamada A, Senda Y, et al. Estimation of the hydromotive source pressure from ejecting beats of the left ventricle. IEEE Trans Biomed Eng 1980; 27: 299-305.

13 Brimioulle S, Wauthy $\mathrm{P}$, Ewalenko $\mathrm{P}$, et al. Single-beat estimation of right ventricular end-systolic pressurevolume relationship. Am J Physiol Heart Circ Physiol 2003; 284: H1625-H1630. 
14 Steen V, Medsger TA Jr. Predictors of isolated pulmonary hypertension in patients with systemic sclerosis and limited cutaneous involvement. Arthritis Rheum 2003; 48: 516-522.

15 Stupi AM, Steen VD, Owens GR, Barnes EL, Rodnan GP, Medsger TA Jr. Pulmonary hypertension in the CREST syndrome variant of systemic sclerosis. Arthritis Rheum 1986; 29: 515-524.

16 Elzinga G, Westerhof N. The effect of an increase in inotropic state and end-diastolic volume on the pumping ability of the feline left heart. Circ Res 1978; 42: 620-628.

17 Elzinga G, Westerhof N. How to quantify pump function of the heart. The value of variables derived from measurements on isolated muscle. Circ Res 1979; 44: 303-308.

18 Suga H, Sagawa K, Shoukas AA. Load independence of the instantaneous pressure-volume ratio of the canine left ventricle and effects of epinephrine and heart rate on the ratio. Circ Res 1973; 32: 314-322.

19 Baan J, van der Velde ET, de Bruin HG, et al. Continuous measurement of left ventricular volume in animals and humans by conductance catheter. Circulation 1984; 70: 812-823.

20 Kuehne T, Yilmaz S, Steendijk P, et al. Magnetic resonance imaging analysis of right ventricular pressure-volume loops: in vivo validation and clinical application in patients with pulmonary hypertension. Circulation 2004; 110: 2010-2016.

21 Follansbee WP, Miller TR, Curtiss EI, et al. A controlled clinicopathologic study of myocardial fibrosis in systemic sclerosis (scleroderma). J Rheumatol 1990; 17: 656-662.

22 Owens GR, Follansbee WP. Cardiopulmonary manifestations of systemic sclerosis. Chest 1987; 91: 118-127.

23 Fernandes F, Ramires FJ, Arteaga E, Ianni BM, Bonfa ES, Mady C. Cardiac remodeling in patients with systemic sclerosis with no signs or symptoms of heart failure: an endomyocardial biopsy study. J Card Fail 2003; 9: 311-317.

24 Westerhof N, Boer C, Lamberts RR, Sipkema P. Cross-talk between cardiac muscle and coronary vasculature. Physiol Rev 2006; 86: 1263-1308.
25 Lamberts RR, Willemsen MJ, Perez NG, Sipkema P, Westerhof N. Acute and specific collagen type I degradation increases diastolic and developed tension in perfused rat papillary muscle. Am J Physiol Heart Circ Physiol 2004; 286: H889-H894.

26 Brower GL, Gardner JD, Forman MF, et al. The relationship between myocardial extracellular matrix remodeling and ventricular function. Eur J Cardiothorac Surg 2006; 30: 604-610.

27 Chanoki M, Ishii M, Kobayashi $\mathrm{H}$, et al. Increased expression of lysyl oxidase in skin with scleroderma. $\mathrm{Br} \mathrm{J}$ Dermatol 1995; 133: 710-715.

28 Brinckmann J, Neess CM, Gaber Y, et al. Different pattern of collagen cross-links in two sclerotic skin diseases: lipodermatosclerosis and circumscribed scleroderma. J Invest Dermatol 2001; 117: 269-273.

29 Bulkley BH, Ridolfi RL, Salyer WR, Hutchins GM. Myocardial lesions of progressive systemic sclerosis. A cause of cardiac dysfunction. Circulation 1976; 53: 483-490.

30 Kahan A, Nitenberg A, Foult JM, et al. Decreased coronary reserve in primary scleroderma myocardial disease. Arthritis Rheum 1985; 28: 637-646.

31 Overbeek MJ, Gan CT, Westerhof N, et al. Cardiac contractility is impaired in systemic sclerosis-associated pulmonary hypertension compared to idiopathic pulmonary hypertension. Am J Respir Crit Care Med 2007; 175: 1002.

32 Meune C, Allanore Y, Devaux JY, et al. High prevalence of right ventricular systolic dysfunction in early systemic sclerosis. J Rheumatol 2004; 31: 1941-1945.

33 Rich S, Dantzker DR, Ayres SM, et al. Primary pulmonary hypertension. A national prospective study. Ann Intern Med 1987; 107: 216-223.

34 Fleg JL, O'Connor F, Gerstenblith G, et al. Impact of age on the cardiovascular response to dynamic upright exercise in healthy men and women. J Appl Physiol 1995; 78: 890-900.

35 Klein AL, Leung DY, Murray RD, Urban LH, Bailey KR, Tajik AJ. Effects of age and physiologic variables on right ventricular filling dynamics in normal subjects. Am J Cardiol 1999; 84: 440-448. 